\title{
Distribution Network Reliability Theoretical Analysis and Assessment Online Considering Load Levels and Power Supply Transferring at Different Period
}

\author{
Huang Zhanhua ${ }^{1}$, Wang Chengmin ${ }^{2}$, Feng Mingcan ${ }^{2}$, Liu Yong ${ }^{3}$, Li \\ Hongzhong ${ }^{4}$ \\ ${ }^{1}$ Shenzhen Power Supply Co.,Ltd., Shenzhen, Guangdong 518001, China \\ ${ }^{2}$ Department of Electrical Engineering, Shanghai Jiao Tong University, Shanghai 200240, China \\ ${ }^{3}$ Shanghai Proinvent Information Tech Ltd, Shanghai 200240, China \\ ${ }^{4}$ College of Electrical Engineering Shanghai University of Electric Power, Shanghai 200090, China
}

Keywords: distribution network; power supply reliability; calculate online; reliability assessment; loop search algorithm

\begin{abstract}
The theory calculation of power supply for distribution network often based on grid structure in planning stage. The period of reliability evaluation indexes are mostly one year. The impact of different load levels and periods are rarely considered. Power supply reliability analysis and calculate online considering different load levels, which has important practical significance. This paper did the research for distribution network reliability online calculation using loop search method. Refining the failure rate of power equipment, outage time, outage users and other information to every period. Making average interruption times of customer (AITC), average interruption hours of customer (AIHC) and reliability on service (RS) as indexes. This method successfully applied in distribution network of Shenzhen reliability management system.
\end{abstract}

\section{Introduction}

The method of power supply reliability evaluate can be divided into two categories as simulation and analytical method [1-4]. Simulation method suitable for complex systems, but it is time-consuming and imprecise. Analytical method is relatively simple. It has been widely used in the distribution system reliability evaluate, which include network equivalent method, minimal path method, traversing fault law, failure mode effects analysis, etc.

Research for the above several algorithms focused on mathematical modeling of the distribution network elements to achieve reliability assessment, and less on the computer implemented method of distribution network reliability especially reliability online calculation studying [5-10]. Network equivalent method and minimum path method need the equivalent of the network, while most of the equivalent procedure by computer are complex to achieve. Fault traverse algorithm is fit for computer calculation. This method calculating reliability indexes of load and system through enumerating each failure element and topology searching affected element. The disadvantage is the low computational efficiency. If you want to find out the element which have the biggest affection to some load point, you need to traverse all elements. Assessment process need to simulate a large number of enumeration failures. Then make the corresponding load transfer analysis. It makes the lack of mutual correlation between each analysis. Many equipment fault can not affect some load point or do not exist available load transfer program. This will cause a lot of load transfer analysis process waste time.

In addition, previous studies on the reliability of the distribution network mainly make the grid structure in the planning stage as the object. Load level often fixed, with little regard for the effect of different load levels to power supply reliability [11-14]. For a running distribution network, online calculation of reliability must consider the level of load and load transfer problems. Because the load level at different times has large difference, and the results of theoretical calculations of reliability 
also have large differences. That is, the reliability online calculation of distribution network closely related to load level and period. The distribution network reliability calculation in the past mainly based on grid structure, rarely considered the difference of load level. Power equipment failure rate does not contain information such as period features.

This paper refines the failure rate of power equipment, outage time, outage users and other information to each period. Making average interruption times of customer (AITC), average interruption hours of customer (AIHC) and reliability on service (RS) as indexes. This method successfully applied in distribution network of Shenzhen reliability management system.

\section{Description of the problem}

The main difference between reliability theoretical calculation online of distribution network reliability and the conventional reliability theoretical calculations is that load level is different at different times. That is, the power supply reliability of distribution network under different load levels will have large difference. Therefore, the reliability data of the device should be detailed classification. That is, power equipment reliability data will be different at different times or under different load levels. Electrical equipment reliability data will be unified put in by different time periods in the database of power production management system (PMS).

A typical distribution network is radial with few loops. As figure 1 trunk divided into three sections with each segment has a branch. For more complex distribution network, the branch also divided into to branch lines. In figure 1 , symbol " $\chi$ " represented breakers, " $\square$ " represented fuses, "__ " represented load switch, "N/O" represented interconnection switch, $\rightarrow$ represented load.

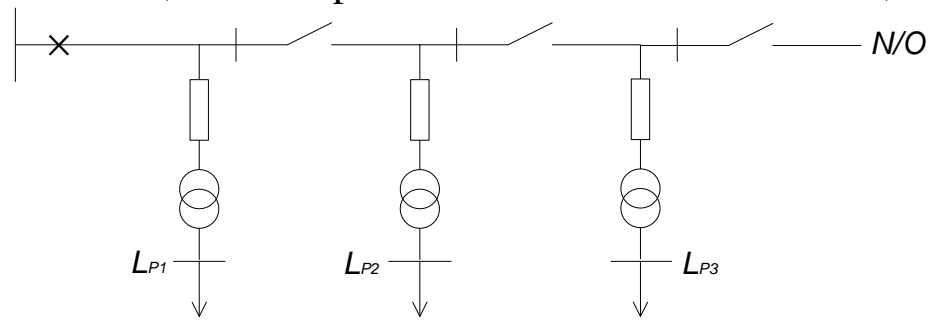

Fig1 A typical distribution network

Here the load characteristic curve is divided into $t=1,2, \mathrm{~L}, T$ typical period. Trunk's failure rate is represented as $\lambda_{l, t}^{m}$. Branch's failure rate is referenced as $\lambda_{l, t}^{m} . l=1,2, \mathrm{~L}, L$ represented the number of segments and the number of branch. Because load points have different loads at different times, in this paper suppose it reflected in the difference of user number. Defined the power of load point $\mathrm{l}$ at $\mathrm{t}$ period is $P_{l, t}$, the number of users on each branch is $N_{l, t}$, and average capacity of per user is $P_{0}$. In other words, the failure rate of electrical equipment has been divided according to the time period. Here we suppose trunk fault repair time is $T^{m}$, branch fault repair time is $T^{d}$.

\section{Loop search method based on load point}

Fundamental. As a typical distribution network has shown in figure 1 has radial network. Under normal circumstances, it can be formed three basic circuits, respectively from power point to load point. As shown below: 


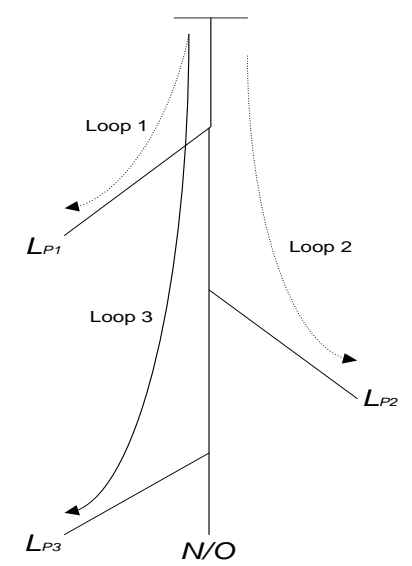

Fig2 Basic loop diagram

The basic idea of loop search method based on the load point is to search for all devices have impact on the load point, and to analyze the impact of outage users and load point outage time due to the location of fault equipment after its failure. As shown above, the equipment which impact load points must be the equipment in appropriate circuit.

For online calculation, the calculation speed is a key issue. Reliability theoretical calculation method which make basic circuit as search target can achieve quickly calculate requirements

Average Interruption Times of Customer (AITC). Average interruption times of customer: customer average interruption frequency during the statistic period, referred to as AITC-1; if excluding external influences, then referred to as AITC-2; if excluding system power shortage, referred to as AITC-3. AITC-3 can use The total number of users can use the cumulative number of user power outages a year divided by the power supply system user number to estimate:

$$
A I T C-3=\frac{\sum_{l} \sum_{t} \lambda_{l, t} N_{l, t}}{\sum_{l} \sum_{t} N_{l, t}}
$$

Wherein, $\lambda_{l, t}$ is the equivalent failure rate of load point $\mathrm{l}$ at $\mathrm{t}$ period. ${ }^{N_{l, t}}$ is the total user number of load point $l$ at t period. So:

$$
\text { AITC }-3=\frac{\sum_{l} \sum_{t} \lambda_{l, t}^{m} \lambda_{l, t}^{d} N_{l, t}}{\sum_{l} \sum_{t} N_{l, t}}
$$

Correspondingly, index AITC-1 and AITC-2 should separately consider the number of power outage users caused by external influences and brownouts.

Average Interruption Hours of Customer (AIHC). Average interruption hours of customer: customer average interruption huors during the statistic period, referred to as AIHC-1; if excluding external influences, then referred to as AIHC-2; if excluding system power shortage, referred to as AIHC-3. AIHC-3 can use The total number of users can use the cumulative hours of user power outages a year divided by the power supply system user number to estimate:

$$
\text { AIHC }-3=\frac{\sum_{l} \sum_{t} N_{l, t}^{\text {off }} T_{l, t}}{\sum_{l} \sum_{t} N_{l, t}}
$$

Wherein, ${ }^{N_{l, t}^{\text {off }}}$ is outage user number of load point l at t period. ${ }^{T_{l, t}}$ is outage time of load point l at $\mathrm{t}$ period. When fault come at trunk, branch or load point $l$ at $\mathrm{t}$ period, it can be divided into three circumstances:

(1) If consider the case of load transfer (fault isolation and restoration of electricity), load point $1,2, \mathrm{~L}, l-1$ after failure resection supply by the original line. Fault duration equals to breakers 
operation time plus load switch operating time (removal of fault zone) plus circuit breaker operation time (recovery of power). Outage time is ignored (because of the repair time is relatively small).

(2) Outage time of load point $l$ depends on the trunk, branch fault repair time:

$$
T_{l, t}=\lambda_{l, t}^{m} T^{m}+\lambda_{l, t}^{d} T^{d}
$$

(3) The load of point $l+1, l+2, \mathrm{~L}, L$ transfer to adjacent feeder lines. Supposing transfer rate is $\gamma_{t}$. The load that have not transferred is $\left(1-\gamma_{t}\right) P_{l, t}$. The outage user number of load point $l+1$ can be calculated as:

$$
N_{l, t}^{\text {off }}=\frac{\left(1-\gamma_{t}\right) P_{l, t}}{P_{0}}
$$

Outage time is:

$$
T_{l, t}=\lambda_{l, t}^{m} T^{m}+\lambda_{l, t}^{d} T^{d}
$$

Then:

$$
\text { AIHC }-3=\frac{\sum_{l} \sum_{t}\left(N_{l, t} T_{l, t}+\sum_{l+1}^{L} N_{l, t}^{\text {off }} T_{l, t}\right)}{\sum_{l} \sum_{t} N_{l, t}}
$$

Correspondingly, index AITC-1 and AITC-2 should separately consider the number of power outage users caused by external influences and brownouts.

Reliability on Service (RS). Reliability on service: During the statistics period, the total time of efficiently supply for users divided by hours of statistics period, referred to as RS-1; if excluding external influences, then referred to as RS-2; if excluding system power shortage, referred to as RS-3. That is:

$$
R S-3=\frac{8760 \sum_{l} \sum_{t} N_{l, t}-\sum_{l} \sum_{t} N_{l, t}^{o f f} T_{l, t}}{8760 \sum_{l} \sum_{t} N_{l, t}} \times 100 \%
$$

Correspondingly, index AITC-1 and AITC-2 should separately consider the number of power outage users caused by external influences and brownouts.

\section{System implementation}

The distribution network reliability analysis method proposed in this paper has successfully applied in distribution network of Shenzhen reliability management system.

Data Platform and Interface. Data interface is critical to realize online real-time calculation of power supply reliability. Shenzhen power supply company building a unified data platform, including topology and device parameters data of distribution network (these data came from distribution GIS management system). It can put out to other system by CIM model which compliance with IEC 61970 standard. CIM data derived from the unified data platform of Shenzhen power supply company includes accurate data status of the grid network structure, equipment connection relation, line type, length, capacity transformers, switches and breaker status. Procession of reading CIM data conversion is shown below. 


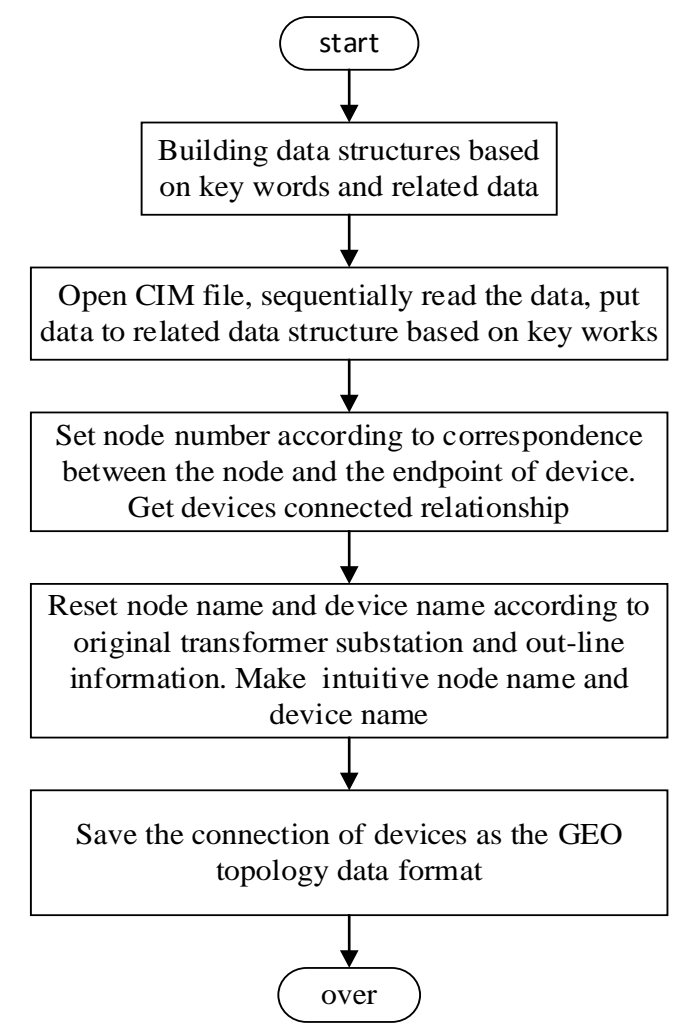

Fig3 CIM structure data conversion stream

Calculation Process of Loop Search Method. Loop search method using recursive algorithm. It greatly reduce programming effort and improve the efficiency of the program. Achieving by sub-loop search and main search loop. As shown below:

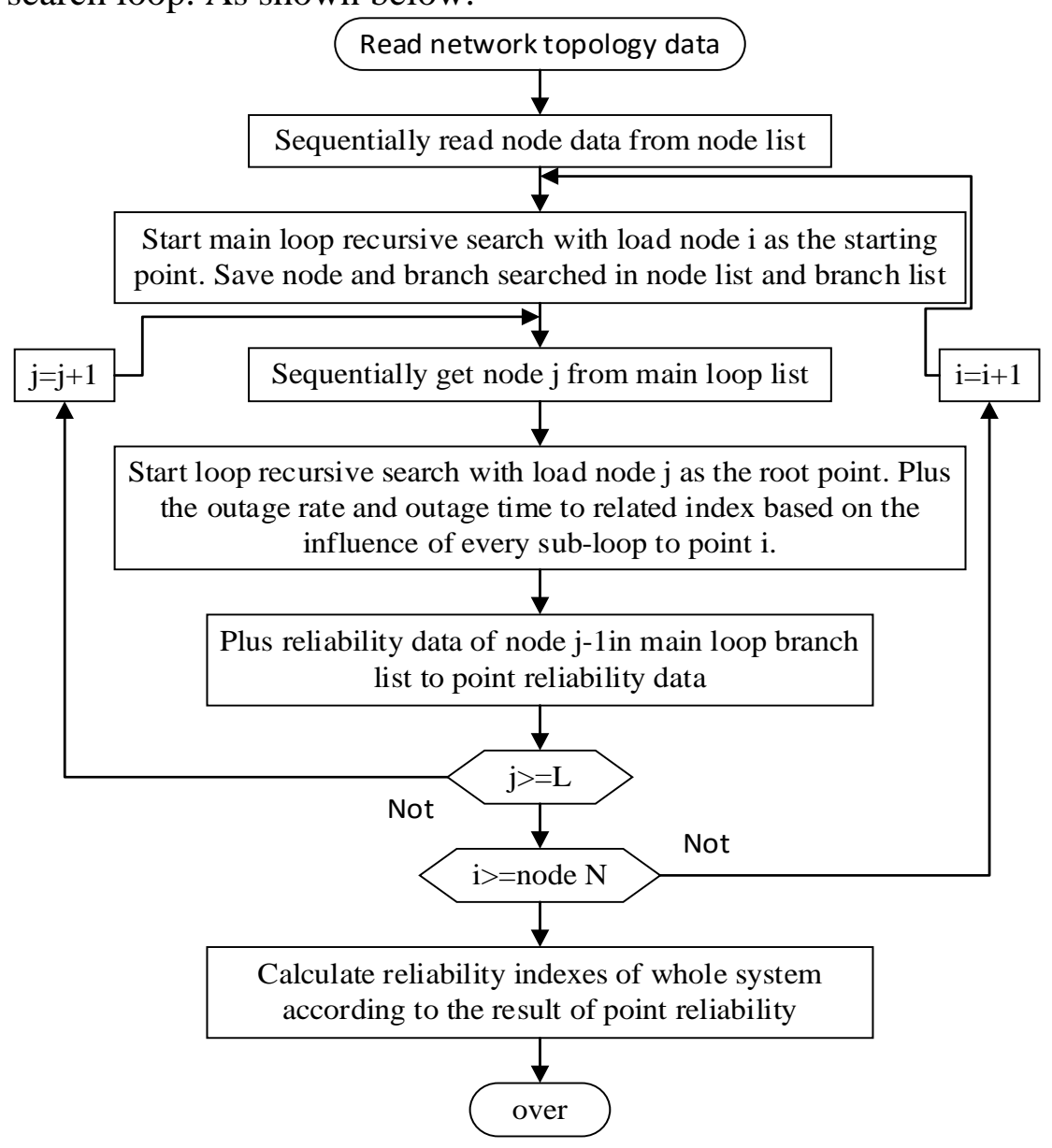

Fig4 Main flow of loop search algorithm 
Reliability Calculation Examples. A single power radiant supply system as the example. Capacity of every line is 6MVA. Power supply radius is $2 \mathrm{~km}$. Let distribution transformer average load ratio is $50 \%$. Average user number of feeder line is 8 . Average length of trunk line is $2 \mathrm{~km}$. Average number of segments is 2 . Reliability of the calculation results are shown in Table 1.

Table1 the reliability of single supply power system

\begin{tabular}{|l|l|}
\hline Name of index & Index value \\
\hline average interruption times of customer AITC-3 (times/household) & 0.1863 \\
\hline average interruption hours of customer AIHC-3 (hours/household) & 3.1124 \\
\hline reliability on service RS-3 (\%) & 99.9645 \\
\hline
\end{tabular}

The results in Table 1 is closer to the actual operating statistics. This shows that the results of the assessment of the results of the online method is more reasonable and reliable. It has a higher reference value.

\section{Conclusions}

This paper took the loop search method to study online calculation of distribution network reliability. It illustrated the effect of different load levels and times to distribution network reliability is very large. The data such as failure rate of power equipment, outage time and user number must be combined with the load level or period. For this reason, indexes evaluation of power supply reliability for distribution network also need refinement. In order to meet the requirements of rapid calculation of reliability of power supply line, make CIM data interface to production management systems (PMIS), SCADA systems, etc. This method achieved good results in the practical application of the distribution network in Shenzhen.

\section{References}

[1] Billinton R,Billinton J E.Distribution system reliability indices[J].IEEE Trans on Power Delivery,1989,4(1):561-568.

[2]Billinton R,Wang P.Reliability-network-equivalent approach to distribution-system-reliability evaluation[J] . IEE Proceedings of Generation,Transmission and Distribution,1998,145(2):149-153.

[3] Wang P,Billinton R,Goel L.Probability distribution evaluation of distribution system reliability indices using a time sequential simulation technique[C]//2000 Canadian Conference on Electrical and Computer Engineering.Halifax,NS:Sous le patronage de IEEE Canadian Atlantic Section IEEE Canada,2000,2:760-764.

[4] Billinton R , Wang P . Teaching distribution system reliability evaluation using Monte Carlo simulation[J].IEEE Trans on PowerSystems,1999,14(2):397-403.

[5] Zhang Peng,Li Wenyuan.Boundary analysis of distribution reliability and economic assessment[J].IEEE Trans on Power Systems,2010,25(2):714-721.

[6] Conti S, Nicolosi R,Rizzo S A.Generalized systematic approach to assess distribution system reliability with renewable distributed generators and microgrids[J].IEEE Transactions on Power Delivery,2012,27(1):1031-1036.

[7] Kyung S K,McKenzie K J,Liu Y L,et al.A study on applications of energy storage for the wind power operation in power systems[C]//IEEE Power Engineering Society General Meeting.Montreal Quebec,Canada,2006:1-5.

[8] Billinton R B.Reliability considerations in the utilization of wind energy,solar energy and energy storage in electric power systems[C]//International Conference on Probabilistic Methods Applied to Power Systems.Stockholm,Sweden,2006:1-6. 
[9] Eicher S,Rahimo M,Tsyplakov E,et al.4.5 kV press pack IGBT designed for ruggedness and reliability[C]//Industry Applications Conference,2004.39th IAS Annual Meeting.Conference Record of the 2004 IEEE.Seattle:IEEE,2004:1534-1539.

[10] IEEE Std 493-2007 (Revision of IEEE Std 493-1997). IEEE Recommended Practice for the Design of Reliable Industrial andCommercial Power Systems[S].USA,2007.

[11] Sithimolada V,Sauer P W.Facility-level DC vs. typical ac distribution for data centers: A comparative reliability study[C]//TENCON 2010-2010 IEEE Region 10 Conference.Fukuoka:IEEE,2010:2102-2107.

[12] Pratt A,Kumar P,Aldridge T V.Evaluation of $400 \mathrm{~V}$ DC distribution in telco and data centers to improve energy efficiency [C]//Telecommunications Energy Conference.INTELEC 2007.29th International.Rome:IEEE,2007:32-39.

[13] Bennett M G,Dhaliwal N S,Leirbukt A.A survey of the reliability of HVDC systems throughout the world during 2009-2010[R].Paris:CIGRE,2012.

[14]Vancers I,Christofersen D J,Leirbukt A,et al.A survey of the reliability of HVDC systems throughout the world during 1999-2000[R].Paris:CIGRE,2002. 